POS $\quad$ PROCEEDINGS

\title{
MAGIC as a Neutrino Follow-Up Instrument
}

\section{A. Fattorini* ${ }^{1}$, K. Satalecka ${ }^{2}$, E. Bernardini ${ }^{3}$, M. Ribó $^{4}$ for the MAGIC Collaboration}

1) Technische Universität Dortmund, Emitl-Figge-Straße 4a, Dortmund, Germany

2) Deutsches Elektronen-Synchrotron (DESY) Zeuthen, Zeuthen, Germany

3) Università degli studi di Padova, Padova, Italy

4) Universitat de Barcelona, ICCUB, IEEC-UB, Barcelona, Spain

E-mail: alicia.fattorinietu-dortmund.de

Since 2012, the stereoscopic IACT system MAGIC, located on La Palma, Canary Islands, has been involved in neutrino follow-up campaigns. The MAGIC telescopes are sensitive to gamma events with energies between $30 \mathrm{GeV}$ and tens of TeV. In 2017 MAGIC detected for the first time very high energy gamma-ray emission from the blazar TXS 0506+056, spatially and temporally correlated with a high-energy neutrino event observed by IceCube. Every time a potentially astrophysical neutrino is detected by IceCube, an alert with the reconstructed coordinates is published. MAGIC uses its automated alert response system and performs follow-up observations in search of a correlated gamma-ray flux. The reconstructed neutrino direction is given with an uncertainty, typically about $0.2^{\circ}-1^{\circ}$. Since the MAGIC angular resolution is much smaller, the analysis needs to be modified to detect sources in a certain sky region. Here we present a method for creating sky maps to identify point sources for the desired sky region, based on a maximum likelihood approach included in the SkyPrism software. Analysis results of the follow-up observation of the neutrino event HESE-160427A performed by MAGIC in April 2016 are shown.

The New Era of Multi-Messenger Astrophysics - Asterics2019

25 - 29 March, 2019

Groningen, The Netherlands

${ }^{*}$ Speaker. 


\section{Multi-messenger Astronomy}

With the first detection of high energy, most likely astrophysical neutrinos at the IceCube observatory in 2013 [1], a new era of multi-messenger astronomy began. In order to investigate the origin of cosmic neutrinos and search for counterparts with other detectors, a worldwide network with an alert system was founded. Among others, the MAGIC Telescopes are involved in follow-up campaigns to search for a counterpart in the very high energy gamma ray band.

\subsection{The Instruments}

The Major Atmospheric Gamma-ray Imaging Cherenkov (MAGIC) Telescopes form a stereoscopic Imaging Air Cherenkov Telescope system, located at the Canary Island La Palma at $2200 \mathrm{~m}$ above sea level. The detector is sensitive to gamma photons with energies between $\sim 30 \mathrm{GeV}$ and up to tens of TeVs. The mirror surface of each telescope has a diameter of $17 \mathrm{~m}$, focusing the light to 1039 Photo Multiplier Tubes (PMTs). The field of view of MAGIC is $3.5^{\circ}$, the angular resolution reaches $0.1^{\circ}$. With its lightweight carbon fiber structure, MAGIC was designed to perform extremely fast repositioning maneuver for follow-up observations of transient sources. Nowadays, MAGIC is able to turn $180^{\circ}$ in $27 \mathrm{~s}$ in the fast mode, reaching a high speed of $7^{\circ}$ per second. [2]

With its volume of $1 \mathrm{~km}^{2}$ the IceCube Detector is currently the biggest neutrino detector. It is located at the South Pole and consists of 86 strings with 80 Digital Optical Modules (DOMs) each. The PMTs, installed in the DOMs, measure the cherenkov light, caused by relativistic charged secondary particles, which are produced in neutrino interactions with the Antarctic ice as detector medium. While a general astrophysical neutrino flux has long been detected by IceCube, the point source identification turns out to be extremely challenging, because of the limited angular resolution and the relatively low statistics of likely astrophysical neutrino events. [1]

\subsection{The Alert System}

Since 2012 MAGIC has been involved in neutrino follow-up campaigns. When IceCube detects a likely astrophysical neutrino event, the alert reaches the main data center in Wisconsin, Madison with a delay of $\sim 33 \mathrm{~s}[3]$ after the event. The coordinates and further information are published at the Gamma-ray Coordinates Network (GCN) [4] as well as at the Astrophysical Multimessenger Observatory Network (AMON) [5] to make the alert available for all observatories. The fast data transfer of the network and the high repositioning speed of MAGIC make follow-up observations in the electromagnetic high energy band $\sim 1$ min after a neutrino event possible. Since the first published alert in 2016, around 6 alerts per year are sent out by IceCube.

\section{The SkyPrism Likelihood Analysis}

In the case of neutrino alerts, the reconstructed coordinates of the events are given with a relatively large uncertainty of typically $\sim 1^{\circ}$ at a $90 \%$ confidence level. The main task of the data analysis is the search of a gamma ray source within the given uncertainty region, which is usually covered by the field of view of MAGIC.

SkyPrism is a maximum likelihood approach based analysis tool testing given source models with a likelihood test. Originally the software was written for the analysis of extended gamma ray 
sources, but it can be applied on various cases, like searching for point sources within a certain region. With the assumption of the source properties and the instrument response of MAGIC a model can be created, described by a likelihood function. Among others, the number of gamma events from the source is a free parameter of the likelihood. With a maximum likelihood test the best values for those parameters to decribe the real data are calculated. [6]

\subsection{The Point Source Search}

In the context of point source search with SkyPrism, the neutrino uncertainty region is scanned by performing the maximum likelihood test of the source model on each position of a predefined grid. The resulting test statistic (TS) value gives information about the probability of the existence of a gamma ray source at each specific position. The advantage of this analysis is the minimized computing time and power, since the parameters for the models are calculated in sky maps, so the source model is obtained by adjusting the coordinates of the assumed grid positions (see fig. 1).

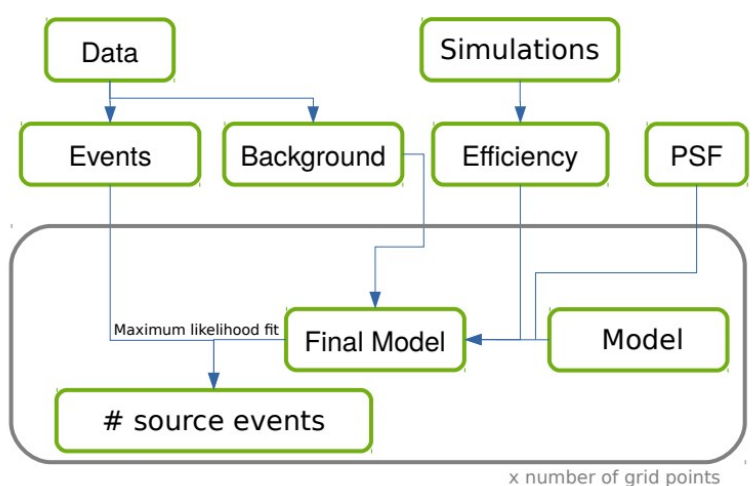

Figure 1: Overview of creating sky maps with SkyPrism maximizing the likelihood function in every grid position of the sky map.

\subsection{Evaluation of the Analysis}

Since SkyPrism is especially tested for sources with known coordinates, additional checks for the case of point source searching within certain regions in the context of follow-up observations are
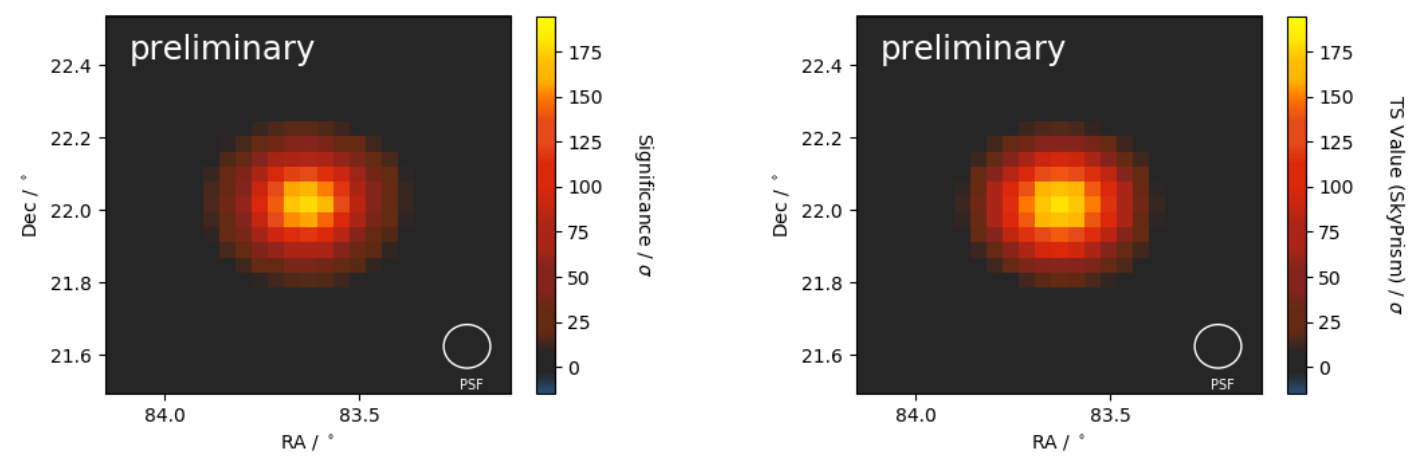

Figure 2: Sky map of the Crab Nebula calculated with the standard software MARS (left) and the sky map produced with SkyPrism (right).

necessary. A reliable way to check a new analysis method is considering a strong and well-known source. The strongest stable source known in the energy range of MAGIC is the Crab Nebula, a pulsar wind nebula in the Milky Way. For the check, a data set of $\sim 16 \mathrm{~h}$ of observation time at low zenith distances $<35^{\circ}$ and good weather conditions is used. As a test for the point source searching following section 2.1, a TS sky map of the Crab Nebula is produced. The standard 


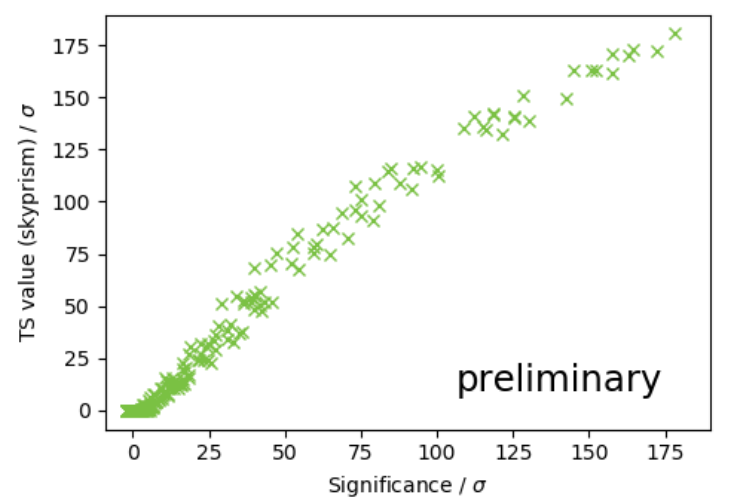

Figure 3: Correlation of significance (MARS) and TS value (SkyPrism) within the sky map. software of MAGIC, called MARS [7], also offers a tool for sky maps, using the Li \& Ma method [8] to calculate the significances of possible sources in a certain sky region. Both sky maps are shown in fig. 2. For the direct comparison, a correlation plot (see fig. 3) is produced. The plot shows a high correlation between the MARS significance and the SkyPrism TS value, which means that both analyses lead to comparable results over the whole sky region and are capable to detect bright sources significantly.

\section{Sources of Neutrino Follow-Up Observations by MAGIC}

In the case of a neutrino alert via the GCN channel, MAGIC uses its automated alert response system, which checks general observation conditions of the reconstructed coordinates at the telescopes site and performs appropriately the followup observation of the puplished position. MAGIC performed six neutrino follow-up observations, marked in fig. 4. The flare of the source TXS 0506+056 detected by MAGIC in 2017 was spatially and temporally correlated with the neutrino event EHE-170922 [9].

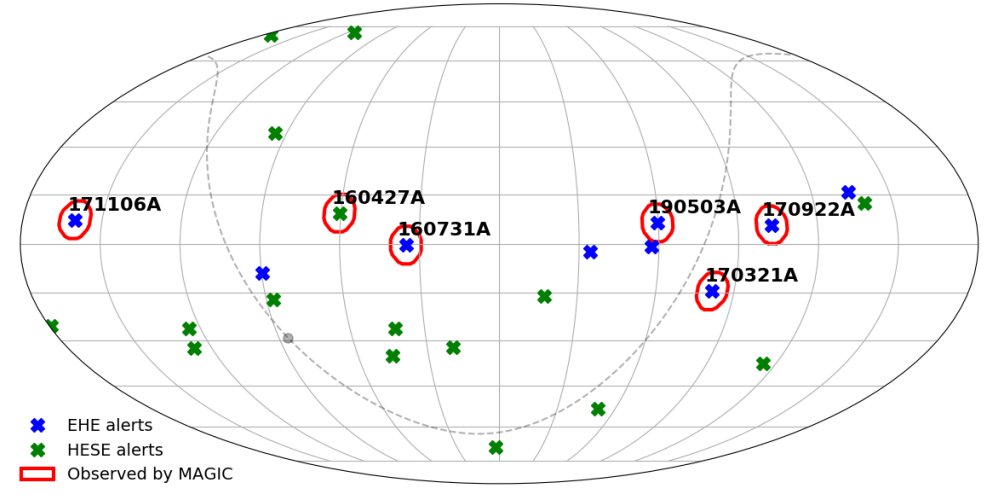

Figure 4: Sky map of the coordinates of all IceCube alerts. [4]

\subsection{Sky Maps produced with the Likelihood Analysis}
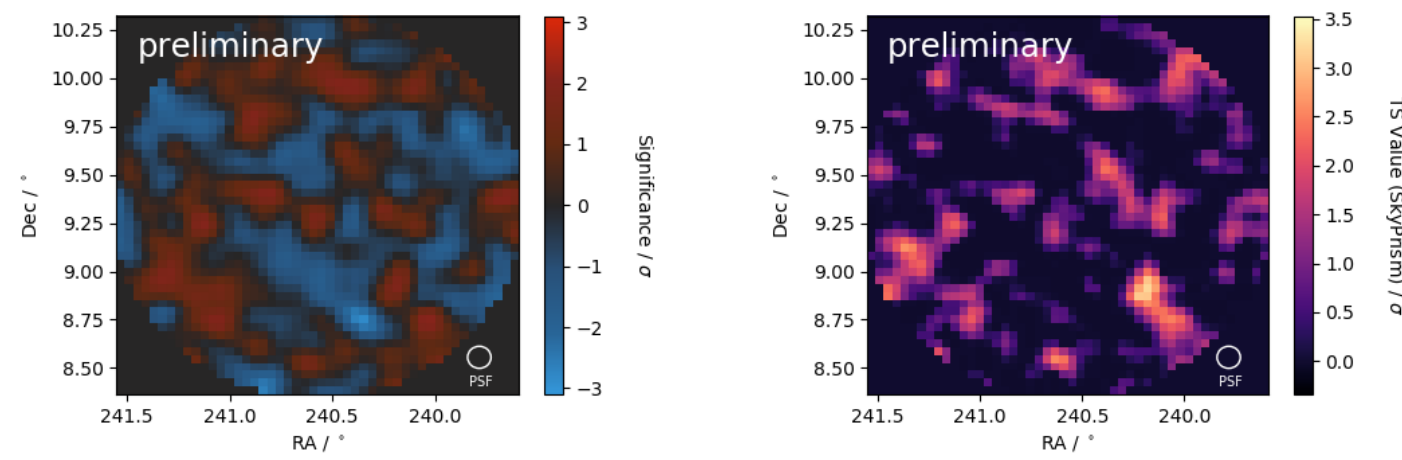

Figure 5: Sky map of the source HESE-160427A calculated with the standard software MARS (left) and the sky map produced with SkyPrism (right). 
The first publication of a neutrino event from the IceCube collaboration via the GCN and AMON channels was on the 27th of April 2016. Because of full moon, MAGIC started the observation with a delay of two days. The measurement was carried out for two hours with moderate moon conditions at low zenith distances $<30^{\circ}$ and clear sky. The sky maps of the SkyPrism and the MARS software are shown in fig. 5. The fluctuations in both maps are comparable. Although none of the sky maps provides the detection of a possible neutrino source within the uncertainty region, the analyses show again consistent results of both methods for point source searching.

\section{Outlook}

In the area of multi-messenger astronomy the analysis methods have to be adapted to new requests. The maximum likelihood method is very suitable for point source searches within the uncertainty region of IceCube follow-up observations. Further tests have to be done to check if a gain in sensitivity can be reached with the maximum likelihood analysis. A further plan is the implementation of flux upper limit calculation and the corresponding TS value with a energy binned analysis to consider the energy dependence of the different parameters. A second goal is to implement the systematic uncertainty of the flux. With the new analysis method for point source searches the MAGIC collaboration will be best prepared for future neutrino alerts.

\section{References}

[1] M. G. Aartsen et al. "The IceCube Neutrino Observatory: Instrumentation and Online Systems". In: JINST 12.03 (2017), P03012.

[2] J. Aleksić et al. "The major upgrade of the MAGIC telescopes, Part II: A performance study using observations of the Crab Nebula". In: Astropart. Phys. 72 (2016), pp. 76-94.

[3] M. G. Aartsen et al. "The IceCube Realtime Alert System”. In: Astropart. Phys. 92 (2017), pp. 30-41.

[4] HEASARC,Astrophysics Science Division (ASD), NASA's GSFC. GCN: The Gamma-ray Coordinates Network. URL: https://gcn.gsfc.nasa.gov/.

[5] M.W.E. Smith et al. "The Astrophysical Multimessenger Observatory Network (AMON)". In: Astroparticle Physics 45 (2013), pp. 56-70.

[6] Ie. Vovk, M. Strzys, and C. Fruck. "Spatial likelihood analysis for MAGIC telescope data - From instrument response modelling to spectral extraction”. In: Astron. Astrophys. 619 (2018), A7.

[7] A. Moralejo et al. "MARS, the MAGIC Analysis and Reconstruction Software". In: arXiv e-prints (July 2009).

[8] T-P Li and Y-Q Ma. "Analysis methods for results in gamma-ray astronomy". In: The Astrophysical Journal 272 (1983), pp. 317-324.

[9] IceCube, Fermi, MAGIC, et al. "Multimessenger observations of a flaring blazar coincident with high-energy neutrino IceCube-170922A”. In: Science 361.6398 (2018), eaat1378. 